\title{
Topographically Distinct Projection Patterns of Early-Generated and Late-Generated Projection Neurons in the Mouse Olfactory Bulb
}

\author{
Uree Chon, ${ }^{1}$ Brandon J. LaFever, ${ }^{2}$ Uyen Nguyen, ${ }^{2}$ Yongsoo Kim, ${ }^{1}$ and Fumiaki Imamura ${ }^{2}$
}

https://doi.org/10.1523/ENEURO.0369-20.2020

${ }^{1}$ Department of Neural and Behavioral Sciences, Penn State College of Medicine, Hershey, PA 17033 and

${ }^{2}$ Department of Pharmacology, Penn State College of Medicine, Hershey, PA 17033

\begin{abstract}
In the mouse brain, olfactory information is transmitted to the olfactory cortex via olfactory bulb (OB) projection neurons known as mitral and tufted cells. Although mitral and tufted cells share many cellular characteristics, these cell types are distinct in their somata location and in their axonal and dendritic projection patterns. Moreover, mitral cells consist of heterogeneous subpopulations. We have previously shown that mitral cells generated at different embryonic days differentially localize within the mitral cell layer (MCL) and extend their lateral dendrites to different sublayers of the external plexiform layer (EPL). Here, we examined the axonal projection patterns from the subpopulations of OB projection neurons that are determined by the timing of neurogenesis (neuronal birthdate) to understand the developmental origin of the diversity in olfactory pathways. We separately labeled early-generated and late-generated OB projection neurons using in utero electroporation performed at embryonic day (E)11 and E12, respectively, and quantitatively analyzed their axonal projection patterns in the whole mouse brain using high-resolution 3D imaging. In this study, we demonstrate that the axonal projection of late-generated $\mathrm{OB}$ projection neurons is restricted to the anterior portion of the olfactory cortex while those of the early-generated OB projection neurons innervate the entire olfactory cortex. Our results suggest that the late-generated mitral cells do not extend their axons to the posterior regions of the olfactory cortex. Therefore, the mitral cells having different birthdates differ, not only in cell body location and dendritic projections within the $\mathrm{OB}$, but also in their axonal projection pattern to the olfactory cortex.
\end{abstract}

Key words: axonal projection; development; mitral cell; neuronal birthdate; olfactory bulb

\section{Significance Statement}

The olfactory bulb $(\mathrm{OB})$ contains long-range projection neurons with distinct connectivity to higher order brain regions. Here, we examined how the birthdate of the OB projection neurons correlates to the generation of differential connectivity patterns. We used in utero electroporation and high-resolution 3D imaging of the whole mouse brain, and determined the topographically distinct axonal projection patterns of early-generated and late-generated $\mathrm{OB}$ projection neurons. Our results show that the timing of neurogenesis is a determining factor for the innervation of $\mathrm{OB}$ projection neurons and indicate that mitral cells having different birthdates are the origins of distinct olfactory information pathways. Our study provides novel insights into the formation of neuronal circuits processing multiple aspects of olfactory information.

Received August 23, 2020; accepted October 16, 2020; First published November 4, 2020.

The authors declare no competing financial interests.
Author contributions: Y.K. and F.I. designed research; U.C., U.N., and F.I. performed research; U.C., B.J.L., Y.K., and F.I. analyzed data; U.C., B.J.L., Y.K., and F.I. wrote the paper. 


\section{Introduction}

The olfactory bulb $(\mathrm{OB})$ is the first relay station for olfactory information in the vertebrate central nervous system. Within the $\mathrm{OB}$, projection neurons, mitral and tufted cells, receive input from olfactory sensory neurons and transmit the olfactory information further to the olfactory cortex consisting of several brain regions. Accumulating evidence suggests that distinct regions within the olfactory cortex process different aspects of the olfactory information. For example, the piriform cortex (PIR) is critical for odor discrimination, identification, and memory (Choi et al., 2011; Wilson and Sullivan, 2011; Bekkers and Suzuki, 2013; Blazing and Franks, 2020), the anterior olfactory nucleus (AON) contributes to odor source detection (Kikuta et al., 2010; Liu et al., 2020), the olfactory tubercle (OT) has close interaction with a reward system (Ikemoto, 2007; Wesson and Wilson, 2011; Gadziola et al., 2015; Yamaguchi, 2017; Zhang et al., 2017), and the amygdala mediates the fear responses induced by predator odors (Root et al., 2014; Isosaka et al., 2015; Kondoh et al., 2016). The segregation of the neural pathways controlling these behavioral responses likely begins with diverse subpopulations of OB projection neurons (Sosulski et al., 2011; Bear et al., 2016).

Historically, the major criterion to discriminate between mitral and tufted cells is somata location within the OB. However, an increasing number of studies have reported differences in the morphologic and physiological properties between these two types of projection neurons in the mammalian OB (Igarashi et al., 2012; Adam et al., 2014; Nagayama et al., 2014b; Cavarretta et al., 2018). In particular, mitral and tufted cells project their axons to distinct regions in the olfactory cortex. While a single mitral cell innervates almost the entire olfactory cortical areas, tufted cells project axons only to the anterior portion of the olfactory cortex, including the OT and AON (Nagayama et al., 2010; Igarashi et al., 2012; Hirata et al., 2019). This suggests that different aspects of olfactory information are processed in parallel pathways originating from mitral and tufted cells. In addition, recent studies have shown that mitral cells consist of heterogeneous subpopulations with different cellular properties. Although mitral cells typically extend their secondary dendrites in the deep sublayer of the external plexiform layer (EPL), some mitral cells extend their secondary dendrites in the superficial sublayer of the EPL (Mori et al., 1983; Orona et al., 1984; Mouradian and Scott, 1988). The diversity of intrinsic biophysical properties among mitral cells, such as interspike interval, firing frequency, and the $I_{h}$ sag current, have also been reported (Nagayama et al., 2004; Padmanabhan and Urban, 2010; Angelo et al., 2012;

This work was supported by National Institutes of Health Grants R01DC016307 (to F.I.) and R01MH116176 (to Y.K.).

Correspondence should be addressed to Fumiaki Imamura at fui1@psu.edu or Yongsoo Kim at yuk17@psu.edu.

https://doi.org/10.1523/ENEURO.0369-20.2020 Copyright @ 2020 Chon et al.

This is an open-access article distributed under the terms of the Creative Commons Attribution 4.0 International license, which permits unrestricted use, distribution and reproduction in any medium provided that the original work is properly attributed.
Igarashi et al., 2012). These differences in molecular and biophysical properties may endow mitral cells with different odor response properties (Dhawale et al., 2010; Kikuta et al., 2013). However, a critical question of whether different subsets of mitral cells project axons to different regions in the olfactory cortex has yet to be answered.

In the developing mouse main $\mathrm{OB}$, mitral cells are generated between embryonic day (E)9 and E13, which is earlier than tufted cell birthdates (Hinds, 1968; Blanchart et al., 2006; Imamura et al., 2011). We previously showed that early-generated and late-generated mitral cells were preferentially localized at the dorsomedial and ventrolateral portion of the mitral cell layer $(\mathrm{MCL})$, respectively (Imamura et al., 2011). Furthermore, we separately labeled subsets of mitral cells with different birthdates using the in utero electroporation method and revealed that early-generated and late-generated mitral cells extend their lateral dendrites in the deep and superficial EPL, respectively, (Imamura and Greer, 2015b). It has been speculated that neuronal birthdates may also control the axonal projection patterns of OB projection neurons to the olfactory cortex (Imamura et al., 2011; Hirata et al., 2019). These previous studies demonstrated that the OT receives axonal inputs preferentially from tufted and late-generated mitral cells (Scott et al., 1980; Imamura et al., 2011), and segregated axonal projections are formed by early-generated mitral cells and late-born external tufted cells (Hirata et al., 2019). Nevertheless, the axonal projection of late-generated mitral cells to the olfactory cortex other than the OT, and differences in axonal projection patterns between early-generated and lategenerated mitral cells have not yet been elucidated. In this study, we separately labeled the early-generated and late-generated $\mathrm{OB}$ projection neurons using the in utero electroporation method and quantitatively analyzed axonal projection patterns in the whole mouse brain using serial two-photon tomography (STPT) imaging. Our study demonstrates that the axonal projection patterns of tufted cells as well as late-generated mitral cells are restricted to the anterior portion of the olfactory cortex.

\section{Materials and Methods}

\section{Animals}

The offspring of CD1 female mice (Charles River; strain code 022; RRID:IMSR_CRL:022) mated with the Tbx21-Cre (B6;CBA-Tg (Tbx21-cre)1Dlc/J; The Jackson Laboratory; stock \#024507; RRID:IMSR_JAX:024507; Haddad et al., 2013) or Tbx21Cre $x$ tdTomato male mice were used for the in utero electroporation in this study. The Tbx21Cre $x$ tdTomato line was created by crossing Tbx21-Cre mice with B6.Cg-Gt(ROSA)26Sortm9 (CAG-tdTomato) Hze/J reporter mice (The Jackson Laboratory; stock \#007909; RRID:IMSR JAX:007909; Nguyen and Imamura, 2019). The day on which we found a copulation plug was called EO, and the succeeding days of gestation were numbered in order. All protocols were approved by, and all methods were performed in accordance with the guidelines of the Institutional Animal Care and Use Committee (IACUC) of Penn State College of Medicine. 


\section{In utero electroporation}

The plasmid that drives the expression of a GFP gene under the CAG promoter in the presence of Cre recombinase (pCALNL-GFP; RRID:Addgene_13770) and the plasmid that expresses tdTomato fluorescent protein under the CAG promoter (pCAG-tdTomato; RRID:Addgene_83029) were obtained from Addgene. In utero electroporation was performed in accordance with the procedure as previously reported (Imamura and Greer, 2013, 2015a). Briefly, pregnant female mice were anesthetized with an intraperitoneal injection of ketamine $(100 \mathrm{mg} / \mathrm{kg})$ and xylazine $(10 \mathrm{mg} / \mathrm{kg})$, and the uterine horns were carefully taken out from the abdominal cavity. Approximately $0.5 \mu \mathrm{l}$ of DNA solution $(1.5-2.5 \mu \mathrm{g} / \mu \mathrm{l}$ in $5 \mathrm{mM}$ Tris- $\mathrm{HCl}(\mathrm{pH} 8.0)$ and $0.5 \mathrm{mM}$ EDTA) was injected into the lateral cerebral ventricle of embryos by insertion of a glass pipette. The DNA solution was mixed with $200 \mu \mathrm{g} / \mathrm{ml}$ of Fast Green for visible confirmation of the injection site. Then, electroporation was conducted by applying square electric pulses: two pulses of $30 \mathrm{~V}, 50-\mathrm{ms}$ duration with a 950-ms interval. To efficiently label the mitral cell precursors in the presumptive $\mathrm{OB}, \mathrm{a}$ positive current was applied from posterior to anterior. Upon completion of the electroporation, the uterine horns were repositioned in the abdominal cavity. Following suturing, the animals were allowed to recover in a warm environment and returned to their home cage. The animals were given a subcutaneous injection of Carprofen $(5 \mathrm{mg} / \mathrm{kg})$ for pain relief before and after the surgery.

\section{Immunohistochemistry}

Postnatal day $(P) 7$ pups were killed by decapitation and fixed in $4 \%$ paraformaldehyde (PFA) overnight. The fixed brains were cryopreserved in $30 \%$ sucrose (wt/ vol) in PBS and embedded in optimal cutting temperature compound (Sakura Finetek USA). The olfactory tissues were cut on a cryostat into $20-\mu \mathrm{m}$ slices, collected on Superfrost Plus Micro Slides (Avantor) and stored at $-80^{\circ} \mathrm{C}$ until use. The slices were pretreated for $30 \mathrm{~min}$ in $0.025 \mathrm{M} \mathrm{HCl}$ at $65^{\circ} \mathrm{C}$ and rinsed with $0.1 \mathrm{M}$ borate buffer ( $\mathrm{pH}$ 8.5), PBS and TBS-T [10 mM Tris- $\mathrm{HCl}(\mathrm{pH}$ 7.4), and $100 \mathrm{~mm} \mathrm{NaCl}$ with $0.3 \%$ Triton X-100 (v/v)]. The slices were then blocked with blocking buffer $[5 \%$ normal donkey serum (v/v) in TBS-T] at $20-25^{\circ} \mathrm{C}$ for $1 \mathrm{~h}$ and incubated with primary antibodies, chicken antiGFP (1:1000; Abcam catalog \#ab13970, RRID:AB 300798) and rabbit anti-tdTomato (1:200; Rockland Immunochemicals catalog \#600-401-379, RRID:AB 2209751), diluted in blocking buffer overnight at $4^{\circ} \mathrm{C}$. Sections were washed with TBS-T and then incubated with secondary antibodies, donkey anti-chicken IgY conjugated with Cy2 (1:200; Jackson ImmunoResearch catalog \#703-225-155, RRID:AB_2340370), and donkey anti-rabbit IgG conjugated with Alexa Fluor 555 (1:300; Thermo Fisher Scientific catalog \#A-31572, RRID:AB 162543), with 4'6-diamino-2-phenylindole dihydrochloride (DAPI; D1306; Thermo Fisher Scientific; RRID:AB_2629482) for nucleus staining for $1 \mathrm{~h}$. The immunoreacted sections were washed and coverslipped with Fluoro-Gel mounting medium (Electron Microscopy Science).

\section{STPT imaging and data analysis}

Mice were transcardially perfused with $0.9 \%$ saline and $4 \%$ PFA. The dissected brains were fixed in $4 \%$ PFA at $4^{\circ}$ $\mathrm{C}$ overnight. These brains were stored in $0.05 \mathrm{M}$ phosphate buffer (PB) at $4^{\circ} \mathrm{C}$ until imaging. Detailed information about STPT imaging and analysis were previously described (Jeong et al., 2016; Newmaster et al., 2020). Briefly, the brain samples were embedded in oxidized $4 \%$ agarose and cross-linked by $0.05 \mathrm{~m}$ sodium borohydride for imaging preparation. This agarose block with an embedded sample was placed in a buffer chamber filled with 0.05 M PB for imaging. We used Tissuecyte 1000 (TissueVision) to perform serial two-photon tomography imaging (Ragan et al., 2012). Each brain was imaged in the coronal plane with a two-photon laser (Coherent Ultrall) at $910 \mathrm{~nm}$ with a $560-\mathrm{nm}$ dichroic mirror to acquire both green and red spectrum signals. Images were acquired as 280 serial sections $(12 \times 16 x y$ tiles, $700 \times 700$ pixels field of view, $1 \times 1 \mu \mathrm{m} x y$ resolution) at every $50 \mu \mathrm{m}$ in thickness. Using a custom-built algorithm, the images were reconstructed and the projection pattern was analyzed. To detect the GFP projection signal, both signal (green) and background (red) images were normalized by z-normalization. Then, the normalized signal channel was subtracted by the normalized background channel. This procedure helped to remove background regardless of the background brightness. Signals from the subtracted images were binarized using a threshold (eight times of SD from the signal channel). The binarized signal was counted in each evenly spaced and non-overlapping rectangular voxel $\left(20 \times 20 \times 50 \mu \mathrm{m}^{3}\right)$ across the whole brain. This procedure helped to quantify the projection area in the brain. Then, each brain with projection signals was registered to Allen common coordinate framework (CCF; Wang et al., 2020) using Elastix (Klein et al., 2010) with previously defined affine and $\mathrm{b}$-spline parameters at $20 \times 20 \times 50 \mu \mathrm{m} x y z$ resolution (Kim et al., 2017).

To quantify the ratio of GFP+ mitral and tufted cells in the main $\mathrm{OB}$, we first selected images of five coronal slices taken every $600 \mu \mathrm{m}$ from anterior to posterior in each OB. Brightness levels were adjusted in Photoshop software (Adobe) to allow for sufficient visualization, but the images were otherwise unaltered. Next, we manually counted all mitral cells classified as GFP + cell bodies in the MCL, and tufted cells classified as GFP + cell bodies in the EPL, in each slice. The ratio of GFP + mitral cells to GFP + tufted cells was calculated by dividing the total number of mitral cells counted from five slices by that of tufted cells. The values were acquired from six OBs (five mice) and eight OBs (seven mice) electroporated at E11 and $\mathrm{E} 12$, respectively.

\section{Olfactory area flatmap}

One OB from each mouse was used to generate a flatmap ( $n=5$ for IUE@E11 and $n=7$ for IUE@E12). First, we generated a maximum projection pattern using the "Add" function on Fiji (ImageJ, NIH) using registered signals onto the reference brain. Then, the lateral olfactory cortex/cortical plate areas with projection signals were selected and exported out using the "TrakEM2" function on 

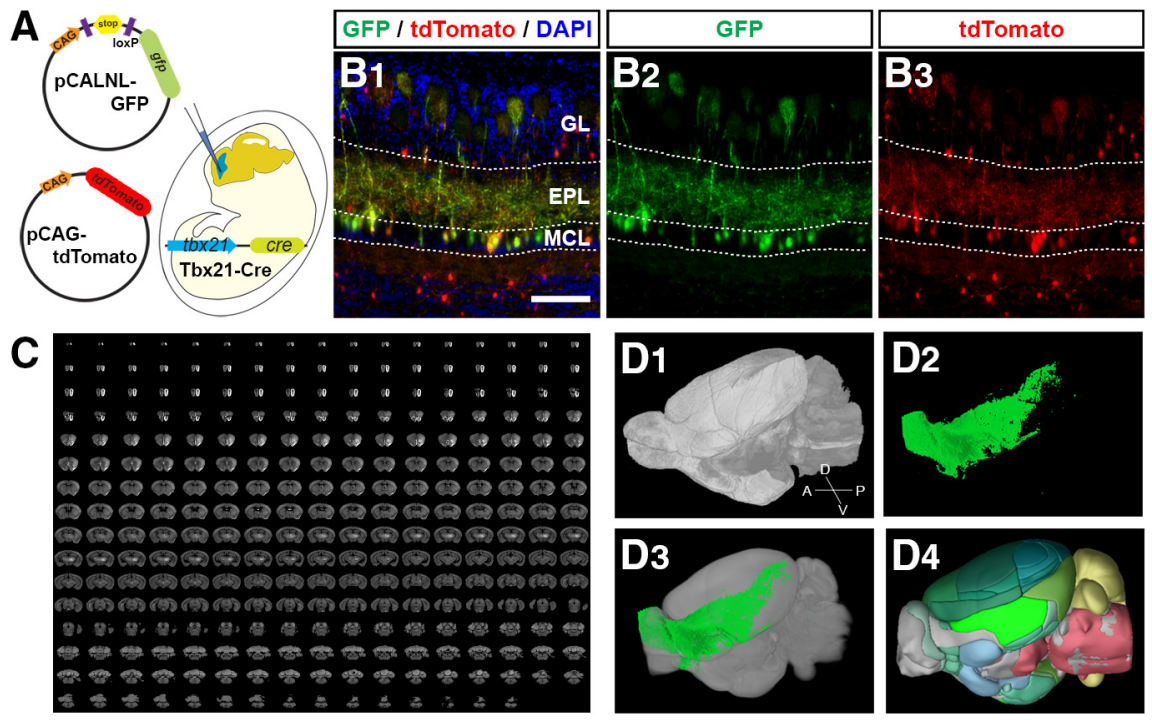

Figure 1. Strategy to analyze the axonal projection patterns of OB projection neurons. $\boldsymbol{A}$, Schematic diagram of in utero electroporation. Plasmid mixture was injected into the lateral ventricle of the mice embryos, and the negative current was applied from posterior to anterior to electroporate the cells in the presumptive OB. B, Medial region of a coronal section of P7 Tbx21-Cre OB electroporated with pCALNL-GFP and pCAG-tdTomato, at E11. OB projection neurons, mitral and tufted cells, express both GFP (green) and tdTomato (red) while tdTomato + interneurons are negative for GFP. All nuclei were stained with DAPI (blue). Scale bar: $100 \mu \mathrm{m}$. C, 270 serial section images acquired in STPT. $\boldsymbol{D}, 3 \mathrm{D}$ reconstruction from the SPTP imaging (D1), axonal projection signal (D2), registered axonal signals in Allen CCF reference brain (D3), and anatomic labels in the reference brain (D4).

Fiji. The exported region was divided into evenly spaced bins to generate a flatmap in the adult reference brain. Each region was given a specific numerical value as a regional ID. To quantify projection signals on the flatmap drawn on the reference brain, GFP signals in each flatmap bin were quantified. Densities of projection signals were measured by counting the numbers of GFP-positive pixels and total pixels in each bin; the quantifications are represented in percentages of GFP-positive pixels. The density was plotted on the flatmap using Excel (Microsoft) and Illustrator (Adobe).

\section{Results}

\section{Electroporation of plasmid vectors to the $\mathrm{OB}$ projection neurons}

We previously showed that in utero electroporation performed at E10 and E12 preferentially labeled earlygenerated and late-generated $\mathrm{OB}$ projection neurons, respectively (Imamura and Greer, 2015b). However, the electroporation also delivers the plasmids into some interneurons in the $\mathrm{OB}$ as well as neurons in the other brain regions including the $\mathrm{AON}, \mathrm{OT}$, and PIR, which makes it difficult to analyze the axonal projection patterns of $\mathrm{OB}$ projection neurons to the olfactory cortex. To overcome this difficulty, we used the Tbx21-Cre transgenic mice in which the Cre recombinase expression is controlled by the Tbx21 promoter (Haddad et al., 2013; Nguyen and Imamura, 2019). Since Tbx21 is exclusively expressed by OB projection neurons in the mouse brain (Mitsui et al., 2011), this method ensures that GFP expression will occur only in $\mathrm{OB}$ projection neurons by electroporating the plasmid, pCALNL-GFP, which expresses
GFP on the presence of Cre recombinase (Fig. 1A). When the pCALNL-GFP and PCAG-tdTomato plasmids are simultaneously electroporated into the Tbx21-Cre mice brain at E11, fluorescent signals of tdTomato were seen in all neuronal cell types, while GFP signals were restricted to the mitral and tufted cells in the OB at P7 (Fig. 1B).

\section{Segregated labeling of $\mathrm{OB}$ projection neurons based on their birthdates}

To compare the axonal projection patterns of OB projection neurons generated at different developmental stages, we electroporated pCALNL-GFP into the brains of Tbx21Cre $x$ tdTomato transgenic mice. In these mice, tdTomato is expressed by all OB projection neurons (Nguyen and Imamura, 2019). Our previous studies showed that differences in cell body location and dendrite extension patterns between E11-generated and E12-generated mitral cells were greater than those between E10generated and E11-generated mitral cells (Imamura et al., 2011; Imamura and Greer, 2015b). We formed the assumption that E12-generated mitral cells significantly change their cellular properties from E11-generated mitral cells. Therefore, we conducted in utero electroporation labeling on E11 (IUE@E11) and E12 (IUE@E12) to examine whether there is a birthdate-dependent difference in the axonal projection patterns. In this experiment, the electroporated mice were killed between six and eightweeks old (P42P53). The GFP signals from the OB projection neurons were examined and analyzed throughout the whole brain at cellular resolution using STPT and custom-built data processing pipeline (for more details, see Materials and Methods; Fig. 1C,D; Jeong et al., 2016). 

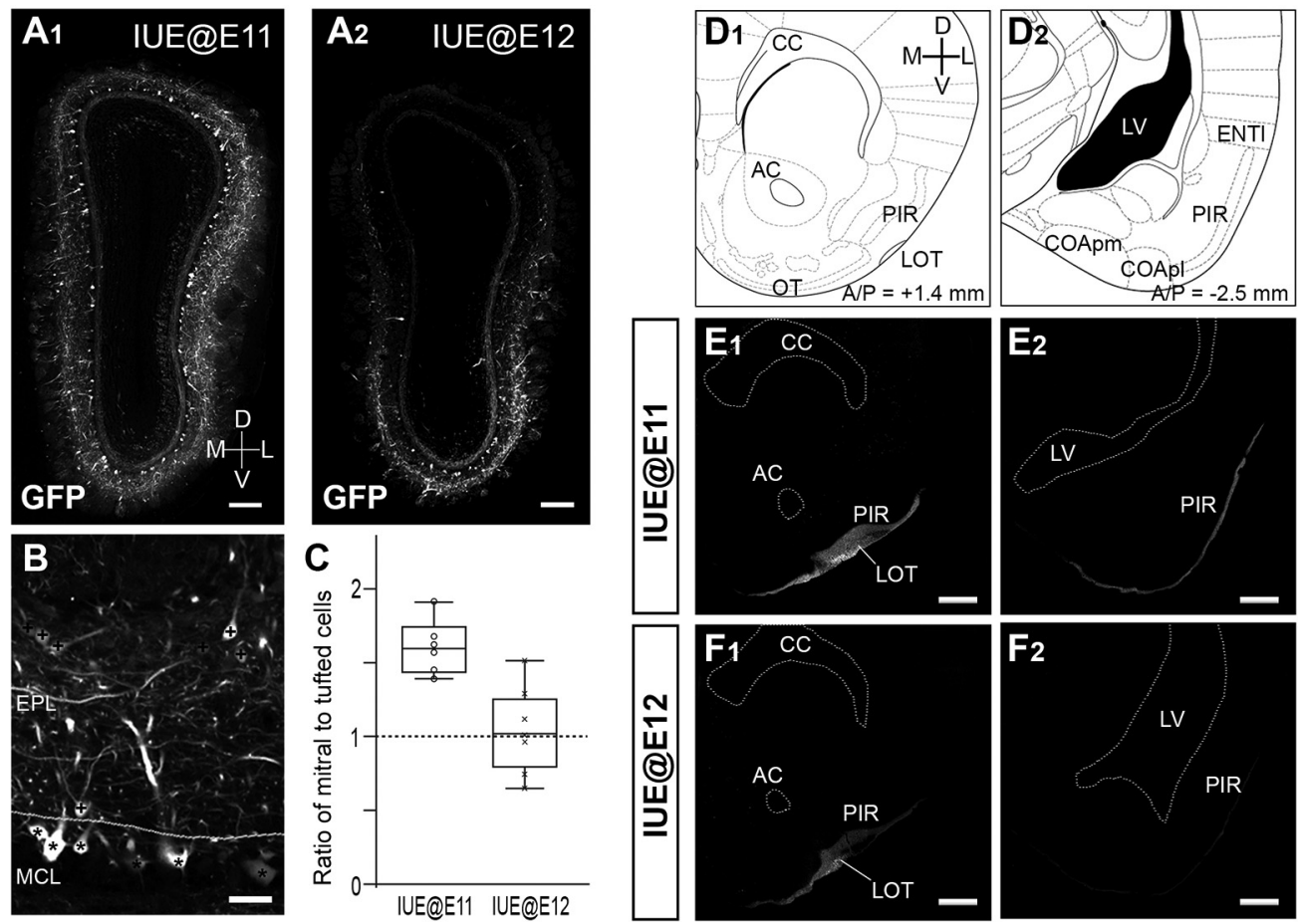

Figure 2. Labeling of different subpopulations of OB projection neurons using in utero electroporation. $\boldsymbol{A}$, Coronal sections of the OBs from adult mice in which electroporations were performed at E11 (A1) or E12 (A2). GFP is expressed only in mitral and tufted cells. IUE@E12 preferentially labeled mitral cells in the ventrolateral part of the OB. B, Quantification of mitral and tufted cells in the OB. Cells that have GFP + somata in the MCL and EPL were defined as mitral cells (marked with asterisks) and tufted cells (marked with plus signs), respectively. $\boldsymbol{C}$, Ratios of mitral cells to tufted cells calculated from IUE@E11 $(n=5)$ and IUE@E12 ( $n=7)$ OBs are shown with box plots. $\boldsymbol{D}-\boldsymbol{F}$, Projection of GFP+ axons to the anterior $(\boldsymbol{D} 1, \boldsymbol{E 1}, \boldsymbol{F 1})$ and posterior $(\boldsymbol{D} 2, \boldsymbol{E 2}, \boldsymbol{F 2})$ part of the olfactory cortex in the IUE@E11 $(\boldsymbol{E})$ and IUE@E12 brain $(\boldsymbol{F})$. Reference brain regions observed in $\boldsymbol{E}, \boldsymbol{F}$ are cited from a mouse brain atlas (Paxinos and Franklin, 2001). GFP+ axons are seen in the anterior PIR of both IUE@E11 (E1) and IUE@E12 (F1) brains, whereas only the IUE@E11 brain has a significant GFP signal in the posterior PIR (E2, F2). Scale bars: $200 \mu \mathrm{m}(\boldsymbol{A}), 50 \mu \mathrm{m}(\boldsymbol{B})$, and $500 \mu \mathrm{m}$ $(\boldsymbol{E}, \boldsymbol{F})$. EPL: external plexiform layer; MCL: mitral cell layer; CC: corpus callosum; AC: anterior commissure; LOT: lateral olfactory tract; PIR: piriform cortex; OT: olfactory tubercle; LV: lateral ventricle; COApl and COApm: posterolateral and posteromedial cortical amygdala; ENTI: lateral entorhinal cortex.

Figure $2 A$ shows the OBs of IUE@E11 and IUE@E12 mice. To examine how the plasmid was taken up between mitral and tufted cells, the number of GFP + mitral cells and tufted cells were counted separately in each OB (Fig. $2 B$ ). Here, we should note that displaced mitral cells, sometimes called internal tufted cells, located at the border of the MCL and EPL were included in the population of mitral cells (Nagayama et al., 2014a). By calculating the ratios of GFP + mitral cells to tufted cells, we confirmed that a significant number of mitral cells was labeled with GFP in the OBs of both IUE@E11 mice $(1.60 \pm 0.13 ; n=6)$ and IUE@E12 mice $(1.05 \pm 0.20 ; n=8)$, although the proportion of labeled mitral cells was lower in the IUE@E12 (Fig. 2C). Of particular note is that GFP+ mitral cells were preferentially found in the ventrolateral $\mathrm{MCL}$ of the IUE@E12 mice whereas the GFP + mitral cells are distributed throughout the whole MCL of the IUE@E11 mice (Fig. 2A). We also confirmed that the GFP+ secondary dendrites were preferentially distributed in the superficial EPL in the IUE@E12 OB. These are consistent findings with our previous study (Imamura and Greer, 2015b) and suggest that, among mitral cells, the late-generated mitral cells were predominantly labeled in the IUE@E12 OB.

\section{Different axonal projection patterns between early- generated and late-generated $\mathrm{OB}$ projection neurons}

Upon imaging the GFP signals in the olfactory cortex, strong signals were observed in the anterior regions, including the lateral olfactory tract (LOT) and the anterior PIR, of both IUE@E11 and IUE@E12 brains (Fig. 2D1,E1, F1). In contrast, IUE@E11 brains showed stronger GFP signals compared with the IUE@E12 brains in the posterior regions of the olfactory cortex, such as the posterior PIR and lateral entorhinal cortex (ENTI; Fig. 2D2,E2,F2). This finding suggests that early-generated OB projection neurons project to broader olfactory cortical areas than the late-generated neurons.

To further analyze the long-range axonal projection patterns of OB projection neurons, GFP signals observed above the threshold level were overlaid onto the coronal sections of a reference brain. Figure $3 A, B$ depicts the distribution of GFP signal in the olfactory cortex from anterior to posterior imaged from a representative IUE@E11 (mitral/tufted ratio=1.45) and IUE@E12 mouse brain (mitral/ tufted ratio $=0.96$; pseudo-colored as red for easy comparison), respectively. In the IUE@E11 brain, the GFP signals were seen in almost every region within the olfactory 

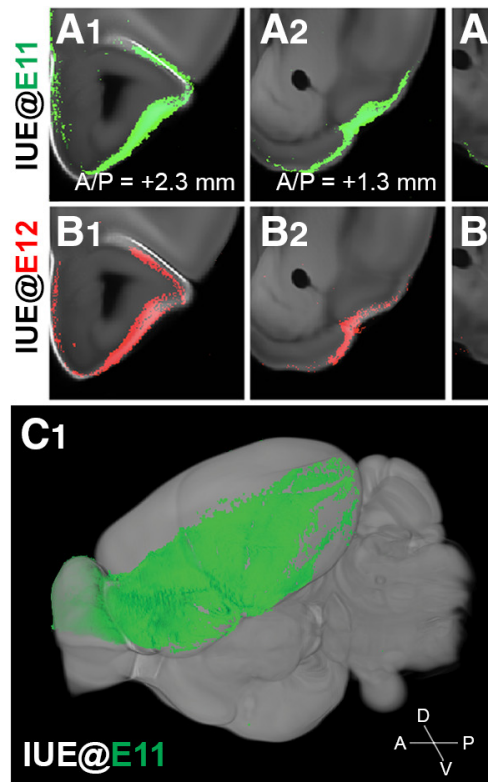
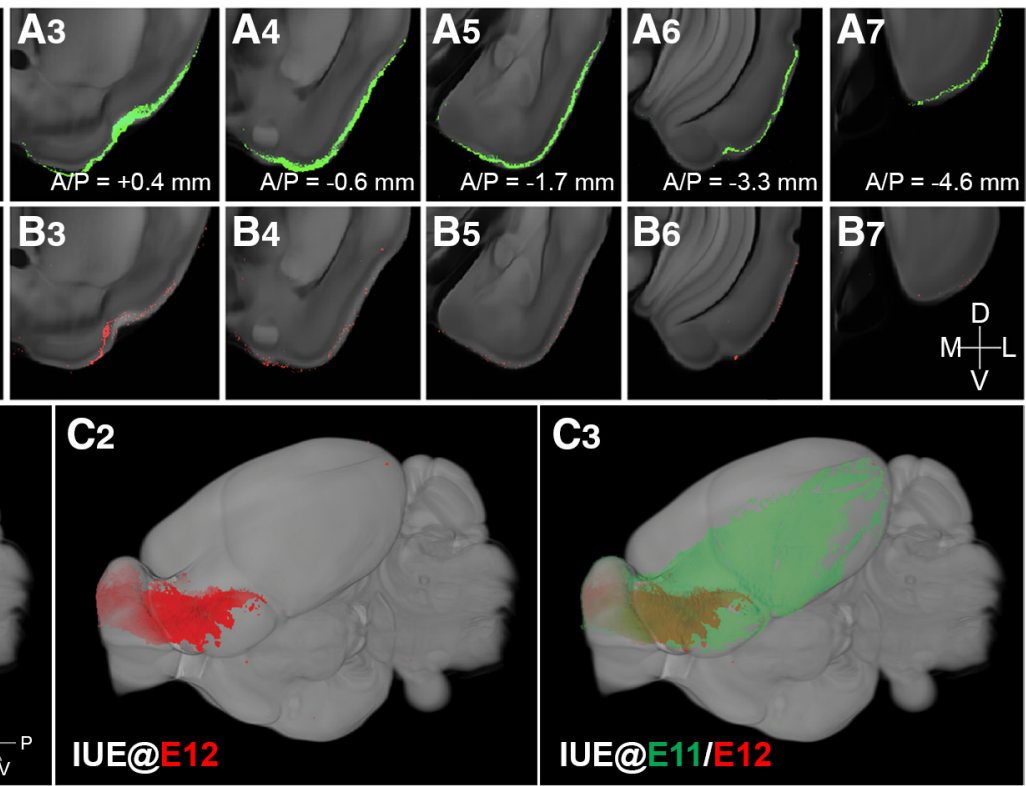

Figure 3. Brain-wide axonal projection pattern from $\mathrm{OB}$ neurons with different birthdates. $\boldsymbol{A}, \boldsymbol{B}$, Axonal projection signals from IUE at E11 $(\boldsymbol{A})$ and IUE at E12 $(\boldsymbol{B})$ registered on the reference brain. GFP signals were pseudo-colored as red in $\boldsymbol{B}$ to facilitate a comparison between signals from two different birth dates. Bregma anterior/posterior (A/P) coordinates were included. $\boldsymbol{C}, 3 D$ rendering of axonal projection from IUE at E11 (C1), E12 (C2), and merged (C3) in the reference brain. Late-generated OB projection neurons labeled with IUE@E12 do not project their axons to the posterior regions of the olfactory cortex.

cortex (Fig. 3A). In contrast, the GFP signal was observed only in the anterior portion of the brain in the IUE@E12 (Fig. 3B). The difference in the distribution of GFP+ axons between IUE@E11 and IUE@E12 brains was clearly displayed when signals from IUE@E11 (green) and IUE@E12 (red; pseudo color) were overlaid onto the reference sections and visualized in a skewed 3D angle (Fig. 3C). These results demonstrate that a subset of $\mathrm{OB}$ projection neurons generated at around E12 restrict their axonal projections solely to the anterior regions of the olfactory cortex.

Next, we devised a digital flatmap of olfactory projection areas (e.g., olfactory cortices) to quantitatively and intuitively visualize the projection patterns. (Fig. $4 A-D$ ). The imaging registration to a common reference brain enabled us to create averaged projection patterns from each IUE@E11 and IUE@E12 brain. Figure 4E,F show the averaged distribution of GFP signals from IUE@E11 $(n=5)$ and IUE@E12 brains $(n=7)$, respectively. The flatmaps clearly indicate that the IUE@E12 brains send little to no projection to the posterior region of the olfactory cortex, such as the posterior PIR, ENTI, and amygdaloid cortex. This reflects the distribution patterns of the individual brain regardless of the numbers of labeled mitral and tufted cells (Fig. 4G,H). Previous studies have shown that the axons of tufted cells primarily project to the AON and OT (Igarashi et al., 2012; Hirata et al., 2019). Moreover, tufted and mitral cells preferentially project to the lateral and medial portion of the OT, respectively. Interestingly, our study shows that the density of GFP+ axons from the IUE@E12 brains, including the axons of late-generated mitral cells as well as those of tufted cells, project mostly to the lateral portion of the OT as compared with the broader projections from the IUE@E11 brains (Fig. 4E,F, regions encircled by white dashed lines). This result suggests that projections from late-generated mitral cells as well as tufted cells primarily innervate the lateral portion of the OT. In addition, the flatmap shows the density gradient of GFP+ axons from anterior to posterior PIR in the IUE@E12 brains (Fig. 4F, regions encircled by yellow dashed lines). The difference between the two groups is highlighted by subtracting the averaged IUE@E12 projection from the averaged IUE@E11 (Fig. 4/). We speculate that $\mathrm{OB}$ projection neurons may gradually shift their axonal endpoint from posterior to anterior within the PIR based on their birthdates.

\section{Discussion}

\section{Topographically distinct projection patterns of early-} generated and late-generated mitral cells

According to a recent study, a single progenitor cell is capable of giving rise to both mitral and tufted cells in the developing OB (Sánchez-Guardado and Lois, 2019). Nevertheless, the generation of mitral cells, which occur between E9-E13, is earlier than that of tufted cells, E11E18 (Hinds, 1968; Hirata et al., 2019). These findings suggest that the timing of neurogenesis is a major determinant for the neuronal properties of $\mathrm{OB}$ projection neurons in the developing brain. Of particular interest is the fact that differences in birthdates among mitral cells or tufted cells result in the generation of OB projection neuron subpopulations with distinct cellular properties (Imamura et al., 2011; Imamura and Greer, 2015b; Hirata et al., 2019). This study demonstrated that the timing of neurogenesis also regulates the axonal projection pattern of different mitral cell subpopulations. 

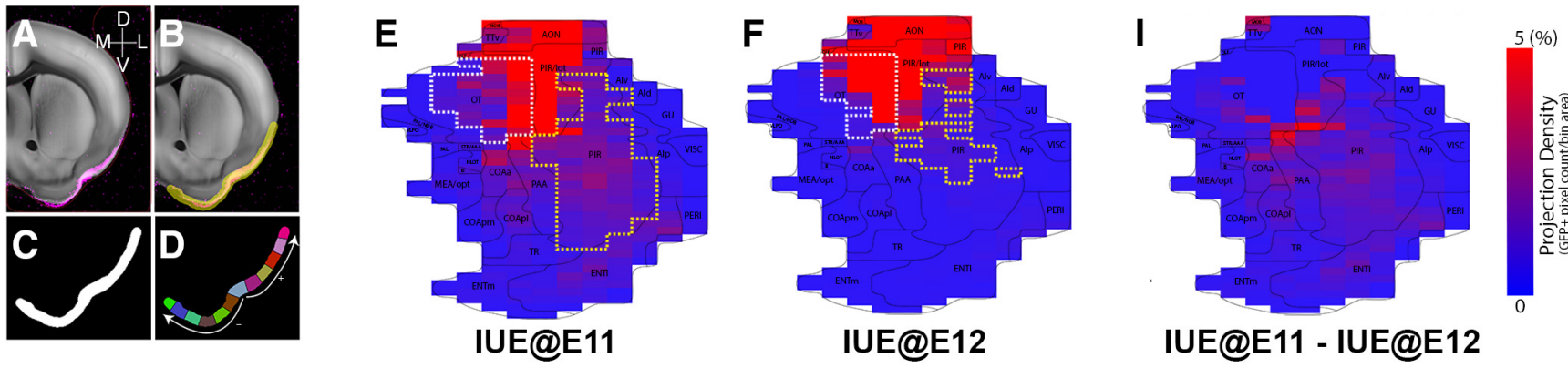

IUE@E11 - IUE@E12

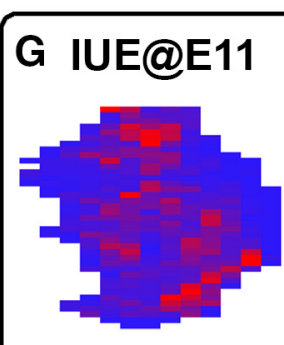

Mitral: 227 cells Tufted: 135 cells (5 OB sections)

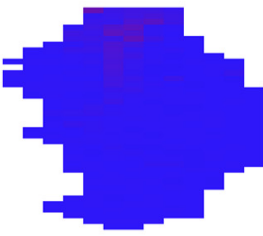

M: 143 cells T: 103 cells

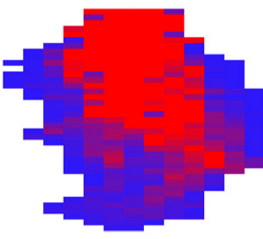

M: 277 cells

T: 191 cells

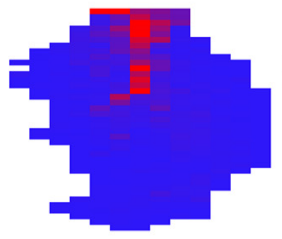

M: 252 cells

T: 161 cells

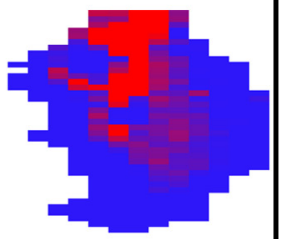

M: 126 cells

T: 66 cells

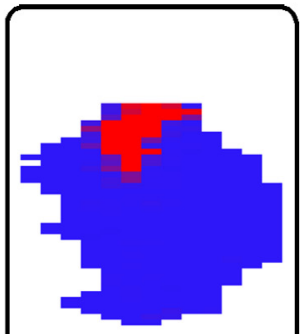

M: 64 cells T: 66 cells

\section{H IUE@E12}

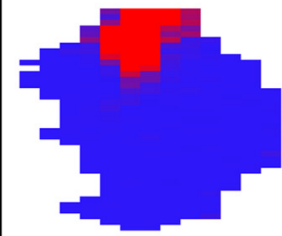

Mitral: 86 cells Tufted: 130 cells (5 OB sections)

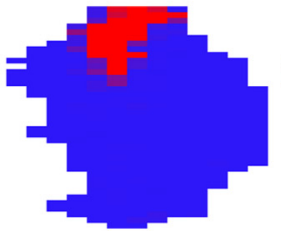

M: 239 cells T: 320 cells

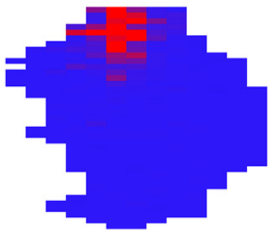

M: 64 cells

T: 62 cells

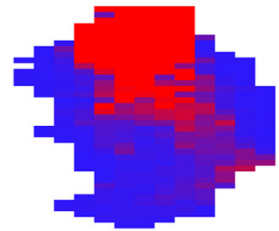

M: 327 cells

T: 215 cells

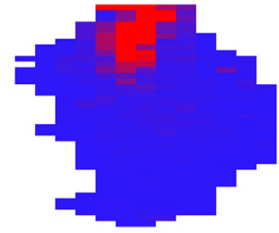

M: 112 cells

T: 110 cells

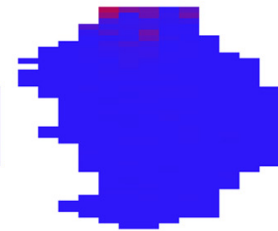

M: 101 cells

T: 90 cells

Figure 4. Topographical axonal projection pattern on 2D flatmap. $\boldsymbol{A}-\boldsymbol{D}$, Creation of 2D flatmap. Axonal projection signal in the reference brain $(\boldsymbol{A})$ and binary mask to cover areas with projection signal $(\boldsymbol{B})$, binary mask $(\boldsymbol{C})$, and evenly spaced bins $(\boldsymbol{D})$ to create the flatmap (for details, see Materials and Methods). E, $\boldsymbol{F}$, Averaged axonal projection signal in heatmap from IUE at E11 (E) and E12 $(\boldsymbol{F})$. Bins that show $>5 \%$ of GFP + signals (projection density) in the OT and PIR are encircled with white and yellow dashed lines, respectively. G, $\boldsymbol{H}$, The 2D flatmaps constructed from five IUE@E11 (G) and seven IUE@E12 (H) individual mouse brains are shown. The numbers of mitral and tufted cells counted from five OB sections are listed under the maps. Dense GFP signals are observed throughout the majority of the olfactory cortex of IUE@E11 brains while only the anterior regions of IUE@E12 brains show dense GFP signals regardless of the numbers of labeled mitral and tufted cells. I, The 2D flatmap in which the averaged IUE@E12 projection $(\boldsymbol{F})$ was subtracted from the averaged IUE@E11 projection $(\boldsymbol{G})$ to highlight the difference between two groups.

Our previous study showed that early-generated and late-generated mitral cell somata preferentially localized in dorsomedial and ventrolateral MCL, respectively (Imamura et al., 2011). Interestingly, the cortical amygdala receives afferent projections preferentially from mitral cells in the dorsomedial MCL (Miyamichi et al., 2011). Our current study demonstrated that late-generated mitral cells do not project to the posterior region of the olfactory cortex, and therefore it is likely that transmission of olfactory information from the dorsomedial $\mathrm{OB}$ to the cortical amygdala is mediated by early-generated mitral cells. This pathway may be essential for the mouse innate fear responses evoked by predator odors (Kobayakawa et al., 2007; Dewan et al., 2013; Root et al., 2014; Isosaka et al., 2015; Kondoh et al., 2016). On the other hand, the OT is innervated by mitral cells in the ventrolateral MCL as well as tufted cells (Scott et al., 1980; Imamura et al., 2011; Igarashi et al., 2012; Hirata et al., 2019). Our study also demonstrated that $O B$ projection neurons generated around E12 innervate the lateral portion of the OT. It has been previously shown that an odor associated with punishment activates the lateral domain of the OT and induces aversive behavior while an odor associated with reward activates the anteromedial domain of the OT and induces attractive behavior (Murata et al., 2015; Yamaguchi, 2017; Zhang et al., 2017). Therefore, neural pathways from the OB to the OT may be mediated by 
distinct populations of $\mathrm{OB}$ projection neurons based on neuronal birthdates; i.e., early-generated $\mathrm{OB}$ projection neurons evoke attractive behavioral responses in mice, whereas late-generated $\mathrm{OB}$ projection neurons are responsible for aversive behaviors. Our study, therefore, suggests that birthdate-dependent mitral cell heterogeneity may be the origins of different olfactory information pathways.

One limitation to our study is that our in utero electroporation technique cannot directly discriminate the axons of late-generated mitral cells from those of tufted cells in the olfactory cortex, and therefore it is possible that late-generated mitral cells do not target the OT. However, we believe this to be unlikely based on our previous study using retrograde Dil labeling of OB projection neurons in which we concluded that mitral cells do innervate the OT (Imamura et al., 2011). This previous study also showed that more E12-generated mitral cells innervated the OT than E10-generated or E11-generated mitral cells. However, it was unknown whether the late-generated mitral cells project their axons to other regions of the olfactory cortex. Our current study clearly demonstrated that the late-generated mitral cells heavily project their axons to the anterior regions of the olfactory cortex, including the OT and AON, but not to the posterior regions. A critical next step is to reveal whether or not the cortical regions innervated by late-generated mitral cells are overlapped with those innervated by tufted cells.

\section{Methods to study the subsets of $\mathrm{OB}$ projection neurons}

The in utero electroporation method has been widely used to label subpopulations of pyramidal neurons in a specific cortical layer as well as a specific type of retinal neurons that are generated at different embryonic days (Stancik et al., 2010; Matsuda, 2015; Bitzenhofer et al., 2017). This method is also effective to separately label OB projection neurons based on their birthdates. We have established an in utero method to target $\mathrm{OB}$ projection neurons and have further shown that the electroporation performed at different embryonic days introduces the plasmids into different subsets of mitral and tufted cells having different birthdates (Imamura and Greer, 2013, 2015b). Here, we performed the electroporation to introduce the GFP plasmids into mouse embryos at E11 and $\mathrm{E} 12$, and found that a significant number of mitral cells were labeled with GFP in the OB regardless of the electroporation timing. Although more GFP+ tufted cells were detected in the OBs following the E12 electroporation as compared with $\mathrm{E} 11$, a consistent finding with our previous study (Imamura and Greer, 2015b), a significant number of mitral cells were also labeled at E12 resulting in a mitral/tufted ratio of almost 1:1. Importantly, the mitral cells labeled with E12 electroporation were mostly the lategenerated mitral cells.

On the other hand, separate labeling of the OB projection neurons having different birthdates has also been successfully accomplished by using a transgenic mouse line expressing CreERT2 under the Neurog2 promoter (Winpenny et al., 2011; Hirata et al., 2019). By altering the timing of tamoxifen injection into the Neurog2CreER $x$
Cdhr1(Pcdh21)tTA x TREtdTomato mouse line (Hirata et al., 2019) induced expression of fluorescent markers in the $\mathrm{OB}$ projection neurons with different birthdates and analyzed their axonal projection patterns. They found that the tufted cells project their axons to the anterior regions of the olfactory cortex and that at least a subpopulation of external tufted cells, the last-generated $\mathrm{OB}$ projection neurons, innervates the anterolateral edge of the OT as well as the pars externa of the AON. However, unlike the previous report showing the enrichment of late-generated mitral cells in the ventrolateral OB (Imamura et al., 2011), the mitral cells labeled within the $\mathrm{OB}$ of this transgenic mouse were distributed in a random manner in the OB regardless of the time of tamoxifen injection. Thus, the in utero electroporation method may be more effective to segregate the early-generated and late-generated mitral cells.

\section{Generation of heterogeneity among $\mathrm{OB}$ projection neurons}

The "canonical" mitral cell typically extends its secondary dendrites throughout the deep portion of the EPL. However, Orona et al. (1984) observed mitral cells with secondary dendrites extending in the intermediate portion of the EPL in the rat OB, although their somata laid in the MCL. Orona et al. (1984) classified mitral cells with secondary dendrites extending throughout the deep or intermediate EPL as Type I and Type II mitral cells, respectively. We have further revealed that early-generated and late-generated mitral cells extend their secondary dendrites in the deep and intermediate EPL, respectively, indicating that late-generated mitral cells can be classified as the previously identified Type II mitral cells (Imamura and Greer, $2015 b)$. Combined with this study, the axonal projection of Type II mitral cells may localize to the more anterior regions of the olfactory cortex. Since the late-generated mitral cells possess the morphologic properties similar to those of tufted cells, an intriguing hypothesis is that the cellular properties of $\mathrm{OB}$ projection neurons are gradually shifted from mitral cells to internal tufted cells followed by middle and external tufted cells. In the developing $\mathrm{OB}$, the progenitor cells may be programmed to produce projection neurons having slightly different properties throughout the course of neurogenesis. This might be a unique feature of the olfactory system since the cellular properties, especially the axonal projection patterns, of cortical pyramidal neurons generated at different timing seems to be less overlapped (Molyneaux et al., 2007; Gerfen et al., 2018).

In order to test the hypothesis that $\mathrm{OB}$ projection neuron diversity is derived from differences in neuronal birthdate, the molecular mechanisms underlying the generation of heterogeneity among the OB projection neurons must first be elucidated. Transcription factors play key roles in determining cellular phenotypes including fate, morphology, and molecular expression profile in developing cerebral pyramidal neurons (Kwan et al., 2012). To date, several transcription factors have been studied in this context with OB projection neurons, such as Tbr1, Tbr2, Neurog1, Neurog2, Sall1, Emx1, Pax6, and AP2 $\varepsilon$ (Yoshida et al., 1997; Bulfone et al., 1998; Arnold et al., 2008; Harrison et al., 2008; Feng 
et al., 2009; Shaker et al., 2012; Imamura and Greer, 2013). Of note, we reported that Tbr1 expression preceded Tbr2 in developing mitral cell (Imamura and Greer, 2013), suggesting that mitral cells follow a non-canonical pathway of differentiation in contrast to that described for cortical pyramidal neurons in which Tbr2 is expressed before Tbr1 during development (Englund et al., 2005). In addition, we and others demonstrated that each transcription factor appears in the developing OB with a distinct spatiotemporal pattern (Williams et al., 2007; Campbell et al., 2011; Nguyen and Imamura, 2019). Thus, comparing the types and time course of transcription factor expression among $\mathrm{OB}$ projection neurons generated at different time points during development is critical to understand the molecular mechanisms underlying the generation of $\mathrm{OB}$ projection neuron diversity. The results from large-scale analyses using omics approaches would help us to advance our knowledge in this field (Campbell et al., 2011; Kawasawa et al., 2016). The in utero electroporation method has the advantage of effectively modifying the molecular functions in a specific subset of mitral/tufted cells by introducing the plasmid vectors, and therefore can be used to study the function of transcription factors responsible for generating the birthdate-dependent differences among mitral cells.

In summary, this study demonstrated that late-generated $\mathrm{OB}$ projection neurons including late-generated mitral cells do not innervate the posterior regions of the olfactory cortex. In addition to somata location and dendritic distribution, our results suggest that the timing of neurogenesis also regulates the axonal projection patterns among OB projection neurons; not only between mitral and tufted cells but also among subpopulations of mitral cells.

\section{References}

Adam Y, Livneh Y, Miyamichi K, Groysman M, Luo L, Mizrahi A (2014) Functional transformations of odor inputs in the mouse olfactory bulb. Front Neural Circuits 8:129.

Angelo K, Rancz EA, Pimentel D, Hundahl C, Hannibal J, Fleischmann A, Pichler B, Margrie TW (2012) A biophysical signature of network affiliation and sensory processing in mitral cells. Nature 488:375-378.

Arnold SJ, Huang GJ, Cheung AF, Era T, Nishikawa S, Bikoff EK, Molnar Z, Robertson EJ, Groszer M (2008) The T-box transcription factor Eomes/Tbr2 regulates neurogenesis in the cortical subventricular zone. Genes Dev 22:2479-2484.

Bear DM, Lassance JM, Hoekstra HE, Datta SR (2016) The evolving neural and genetic architecture of vertebrate olfaction. Curr Biol 26:R1039-R1049.

Bekkers JM, Suzuki N (2013) Neurons and circuits for odor processing in the piriform cortex. Trends Neurosci 36:429-438.

Bitzenhofer SH, Ahlbeck J, Wolff A, Wiegert JS, Gee CE, Oertner TG, Hanganu-Opatz IL (2017) Layer-specific optogenetic activation of pyramidal neurons causes beta-gamma entrainment of neonatal networks. Nat Commun 8:14563.

Blanchart A, De Carlos JA, López-Mascaraque L (2006) Time frame of mitral cell development in the mice olfactory bulb. J Comp Neurol 496:529-543.

Blazing RM, Franks KM (2020) Odor coding in piriform cortex: mechanistic insights into distributed coding. Curr Opin Neurobiol 64:96102.

Bulfone A, Wang F, Hevner R, Anderson S, Cutforth T, Chen S, Meneses J, Pedersen R, Axel R, Rubenstein JL (1998) An olfactory sensory map develops in the absence of normal projection neurons or GABAergic interneurons. Neuron 21:1273-1282.

Campbell GR, Baudhuin A, Vranizan K, Ngai J (2011) Transcription factors expressed in olfactory bulb local progenitor cells revealed by genome-wide transcriptome profiling. Mol Cell Neurosci 46:548-561.

Cavarretta F, Burton SD, Igarashi KM, Shepherd GM, Hines ML, Migliore M (2018) Parallel odor processing by mitral and middle tufted cells in the olfactory bulb. Sci Rep 8:7625.

Choi GB, Stettler DD, Kallman BR, Bhaskar ST, Fleischmann A, Axel $R$ (2011) Driving opposing behaviors with ensembles of piriform neurons. Cell 146:1004-1015.

Dewan A, Pacifico R, Zhan R, Rinberg D, Bozza T (2013) Non-redundant coding of aversive odours in the main olfactory pathway. Nature 497:486-489.

Dhawale AK, Hagiwara A, Bhalla US, Murthy VN, Albeanu DF (2010) Non-redundant odor coding by sister mitral cells revealed by light addressable glomeruli in the mouse. Nat Neurosci 13:1404-1412.

Englund C, Fink A, Lau C, Pham D, Daza RA, Bulfone A, Kowalczyk T, Hevner RF (2005) Pax6, Tbr2, and Tbr1 are expressed sequentially by radial glia, intermediate progenitor cells, and postmitotic neurons in developing neocortex. J Neurosci 25:247-251.

Feng W, Simoes-de-Souza F, Finger TE, Restrepo D, Williams T (2009) Disorganized olfactory bulb lamination in mice deficient for transcription factor AP-2epsilon. Mol Cell Neurosci 42:161-171.

Gadziola MA, Tylicki KA, Christian DL, Wesson DW (2015) The olfactory tubercle encodes odor valence in behaving mice. $\mathrm{J}$ Neurosci 35:4515-4527.

Gerfen CR, Economo MN, Chandrashekar J (2018) Long distance projections of cortical pyramidal neurons. J Neurosci Res 96:1467-1475.

Haddad R, Lanjuin A, Madisen L, Zeng H, Murthy VN, Uchida N (2013) Olfactory cortical neurons read out a relative time code in the olfactory bulb. Nat Neurosci 16:949-957.

Harrison SJ, Nishinakamura R, Monaghan AP (2008) Sall1 regulates mitral cell development and olfactory nerve extension in the developing olfactory bulb. Cereb Cortex 18:1604-1617.

Hinds JW (1968) Autoradiographic study of histogenesis in the mouse olfactory bulb. I. Time of origin of neurons and neuroglia. $J$ Comp Neurol 134:287-304.

Hirata T, Shioi G, Abe T, Kiyonari H, Kato S, Kobayashi K, Mori K, Kawasaki T (2019) A novel birthdate-labeling method reveals segregated parallel projections of mitral and external tufted cells in the main olfactory system. eNeuro 6:ENEURO.0234-19.2019.

Igarashi KM, leki N, An M, Yamaguchi Y, Nagayama S, Kobayakawa K, Kobayakawa R, Tanifuji M, Sakano H, Chen WR, Mori K (2012) Parallel mitral and tufted cell pathways route distinct odor information to different targets in the olfactory cortex. J Neurosci 32:7970-7985

Ikemoto S (2007) Dopamine reward circuitry: two projection systems from the ventral midbrain to the nucleus accumbens-olfactory tubercle complex. Brain Res Rev 56:27-78.

Imamura F, Greer CA (2013) Pax6 regulates Tbr1 and Tbr2 expressions in olfactory bulb mitral cells. Mol Cell Neurosci 54:58-70.

Imamura F, Greer CA (2015a) Electroporation in the developing mouse olfactory bulb. In: Electroporation methods and neuroscience, neuromethods (Saito T, ed), pp 69-79. New York: Springer Science+Business Media, LLC.

Imamura F, Greer CA (2015b) Segregated labeling of olfactory bulb projection neurons based on their birthdates. Eur $\mathrm{J}$ Neurosci 41:147-156.

Imamura F, Ayoub AE, Rakic P, Greer CA (2011) Timing of neurogenesis is a determinant of olfactory circuitry. Nat Neurosci 14:331337.

Isosaka T, Matsuo T, Yamaguchi T, Funabiki K, Nakanishi S, Kobayakawa R, Kobayakawa K (2015) Htr2a-expressing cells in the central amygdala control the hierarchy between innate and learned fear. Cell 163:1153-1164. 
Jeong M, Kim Y, Kim J, Ferrante DD, Mitra PP, Osten P, Kim D (2016) Comparative three-dimensional connectome map of motor cortical projections in the mouse brain. Sci Rep 6:20072.

Kawasawa YI, Salzberg AC, Li M, Sestan N, Greer CA, Imamura F (2016) RNA-seq analysis of developing olfactory bulb projection neurons. Mol Cell Neurosci 74:78-86.

Kikuta S, Sato K, Kashiwadani H, Tsunoda K, Yamasoba T, Mori K (2010) Neurons in the anterior olfactory nucleus pars externa detect right or left localization of odor sources. Proc Natl Acad Sci USA 107:12363-12368.

Kikuta S, Fletcher ML, Homma R, Yamasoba T, Nagayama S (2013) Odorant response properties of individual neurons in an olfactory glomerular module. Neuron 77:1122-1135.

Kim Y, Yang GR, Pradhan K, Venkataraju KU, Bota M, García Del Molino LC, Fitzgerald G, Ram K, He M, Levine JM, Mitra P, Huang ZJ, Wang XJ, Osten P (2017) Brain-wide maps reveal stereotyped cell-type-based cortical architecture and subcortical sexual dimorphism. Cell 171:456-469.e22.

Klein S, Staring M, Murphy K, Viergever MA, Pluim JP (2010) Elastix: a toolbox for intensity-based medical image registration. IEEE Trans Med Imaging 29:196-205.

Kobayakawa K, Kobayakawa R, Matsumoto H, Oka Y, Imai T, Ikawa M, Okabe M, Ikeda T, Itohara S, Kikusui T, Mori K, Sakano H (2007) Innate versus learned odour processing in the mouse olfactory bulb. Nature 450:503-508.

Kondoh K, Lu Z, Ye X, Olson DP, Lowell BB, Buck LB (2016) A specific area of olfactory cortex involved in stress hormone responses to predator odours. Nature 532:103-106.

Kwan KY, Sestan N, Anton ES (2012) Transcriptional co-regulation of neuronal migration and laminar identity in the neocortex. Development 139:1535-1546.

Liu A, Papale AE, Hengenius J, Patel K, Ermentrout B, Urban NN (2020) Mouse navigation strategies for odor source localization. Front Neurosci 14:218.

Matsuda T (2015) Electroporation in the rodent retina in vivo and in vitro. In: Electroporation methods and neuroscience, neuromethods (Saito T, ed), pp 47-67. New York: Springer Science+Business Media, LLC.

Mitsui S, Igarashi KM, Mori K, Yoshihara Y (2011) Genetic visualization of the secondary olfactory pathway in Tbx21 transgenic mice. Neural Syst Circuits 1:5.

Miyamichi K, Amat F, Moussavi F, Wang C, Wickersham I, Wall NR, Taniguchi H, Tasic B, Huang ZJ, He Z, Callaway EM, Horowitz MA, Luo $L$ (2011) Cortical representations of olfactory input by transsynaptic tracing. Nature 472:191-196.

Molyneaux BJ, Arlotta P, Menezes JR, Macklis JD (2007) Neuronal subtype specification in the cerebral cortex. Nat Rev Neurosci 8:427-437.

Mori K, Kishi K, Ojima H (1983) Distribution of dendrites of mitral, displaced mitral, tufted, and granule cells in the rabbit olfactory bulb. J Comp Neurol 219:339-355.

Mouradian LE, Scott JW (1988) Cytochrome oxidase staining marks dendritic zones of the rat olfactory bulb external plexiform layer. $J$ Comp Neurol 271:507-518.

Murata K, Kanno M, leki N, Mori K, Yamaguchi M (2015) Mapping of Learned Odor-Induced Motivated Behaviors in the Mouse Olfactory Tubercle. J Neurosci 35:10581-10599.

Nagayama S, Takahashi YK, Yoshihara Y, Mori K (2004) Mitral and tufted cells differ in the decoding manner of odor maps in the rat olfactory bulb. J Neurophysiol 91:2532-2540.

Nagayama S, Enerva A, Fletcher ML, Masurkar AV, Igarashi KM, Mori K, Chen WR (2010) Differential axonal projection of mitral and tufted cells in the mouse main olfactory system. Front Neural Circuits 4:120.

Nagayama S, Homma R, Imamura F (2014a) Neuronal organization of olfactory bulb circuits. Front Neural Circuits 8:98.
Nagayama S, Igarashi KM, Manabe H, Mori K (2014b) Parallel tufted cell and mitral cell pathways from the olfactory bulb to the olfactory cortex. In: The olfactory system (Mori K, ed). Tokyo: Springer.

Newmaster KT, Nolan ZT, Chon U, Vanselow DJ, Weit AR, Tabbaa M, Hidema S, Nishimori K, Hammock EAD, Kim Y (2020) Quantitative cellular-resolution map of the oxytocin receptor in postnatally developing mouse brains. Nat Commun 11:1885.

Nguyen UP, Imamura F (2019) Regional differences in mitral cell development in mouse olfactory bulb. J Comp Neurol 527:2233-2244.

Orona E, Rainer EC, Scott JW (1984) Dendritic and axonal organization of mitral and tufted cells in the rat olfactory bulb. J Comp Neurol 226:346-356.

Padmanabhan K, Urban NN (2010) Intrinsic biophysical diversity decorrelates neuronal firing while increasing information content. Nat Neurosci 13:1276-1282.

Paxinos G, Franklin KBJ (2001) The mouse brain in stereotaxic coordinates. San Diego: Academic Press.

Ragan T, Kadiri LR, Venkataraju KU, Bahlmann K, Sutin J, Taranda J, Arganda-Carreras I, Kim Y, Seung HS, Osten P (2012) Serial twophoton tomography for automated ex vivo mouse brain imaging. Nat Methods 9:255-258.

Root CM, Denny CA, Hen R, Axel R (2014) The participation of cortical amygdala in innate, odour-driven behaviour. Nature 515:269-273.

Sánchez-Guardado L, Lois C (2019) Lineage does not regulate the sensory synaptic input of projection neurons in the mouse olfactory bulb. Elife 8:e46675.

Scott JW, McBride RL, Schneider SP (1980) The organization of projections from the olfactory bulb to the piriform cortex and olfactory tubercle in the rat. J Comp Neurol 194:519-534.

Shaker T, Dennis D, Kurrasch DM, Schuurmans C (2012) Neurog1 and Neurog2 coordinately regulate development of the olfactory system. Neural Dev 7:28.

Sosulski DL, Lissitsyna Bloom M, Cutforth T, Axel R, Datta SR (2011) Distinct representations of olfactory information in different cortical centres. Nature 472:213-216.

Stancik EK, Navarro-Quiroga I, Sellke R, Haydar TF (2010) Heterogeneity in ventricular zone neural precursors contributes to neuronal fate diversity in the postnatal neocortex. J Neurosci 30:7028-7036.

Wang Q, Ding SL, Li Y, Royall J, Feng D, Lesnar P, Graddis N, Naeemi M, Facer B, Ho A, Dolbeare T, Blanchard B, Dee N, Wakeman W, Hirokawa KE, Szafer A, Sunkin SM, Oh SW, Bernard A, Phillips JW, et al. (2020) The Allen mouse brain common coordinate framework: a 3D reference atlas. Cell 181:936-953.e20.

Wesson DW, Wilson DA (2011) Sniffing out the contributions of the olfactory tubercle to the sense of smell: hedonics, sensory integration, and more? Neurosci Biobehav Rev 35:655-668.

Williams EO, Xiao Y, Sickles HM, Shafer P, Yona G, Yang JY, Lin DM (2007) Novel subdomains of the mouse olfactory bulb defined by molecular heterogeneity in the nascent external plexiform and glomerular layers. BMC Dev Biol 7:48.

Wilson DA, Sullivan RM (2011) Cortical processing of odor objects. Neuron 72:506-519.

Winpenny E, Lebel-Potter M, Fernandez ME, Brill MS, Götz M, Guillemot F, Raineteau O (2011) Sequential generation of olfactory bulb glutamatergic neurons by Neurog2-expressing precursor cells. Neural Dev 6:12.

Yamaguchi M (2017) Functional sub-circuits of the olfactory system viewed from the olfactory bulb and the olfactory tubercle. Front Neuroanat 11:33.

Yoshida M, Suda Y, Matsuo I, Miyamoto N, Takeda N, Kuratani S, Aizawa S (1997) Emx1 and Emx2 functions in development of dorsal telencephalon. Development 124:101-111.

Zhang Z, Liu Q, Wen P, Zhang J, Rao X, Zhou Z, Zhang H, He X, Li J, Zhou Z, Xu X, Zhang X, Luo R, Lv G, Li H, Cao P, Wang L, Xu F (2017) Activation of the dopaminergic pathway from VTA to the medial olfactory tubercle generates odor-preference and reward. Elife 6:e25423. 